\title{
MiR-124 suppresses tumor growth and metastasis by targeting Foxq1 in nasopharyngeal carcinoma
}

\author{
Xiao Hong Peng ${ }^{1 \dagger}$, Hao Ran Huang ${ }^{1 \dagger}$, Juan Lu ${ }^{1 \dagger}$, Xiong Liu', Fei Peng Zhao ${ }^{1}$, Bao Zhang ${ }^{2}$, Shao Xiong Lin ${ }^{1,3}$, \\ Lu Wang ${ }^{1}$, Huai Hong Chen ${ }^{1}$, Xia Xu', Fan Wang ${ }^{1}$ and Xiang Ping Li ${ }^{1 *}$
}

\begin{abstract}
Background: The molecular mechanisms underlying dysregulation of microRNAs have been documented in nasopharyngeal carcinoma (NPC). Our previous study demonstrated that plasma miR-124 was down-regulated in NPC using microarray analysis and quantitative PCR validation. Though growing studies showed that down-regulated miR-124 was closely related to tumourigenesis in various types of cancers, the role of miR-124 in NPC remains largely unknown.
\end{abstract}

Methods: The expression level of miR-124 was evaluated in NPC cell lines and patient specimens using quantitative reverse transcription-PCR (Real-time qPCR). The clinicopathological significance of the resultant data was later analyzed. Then, we explored the role of miR-124 in NPC tumorigenesis by in vitro and in vivo experiments. Homo sapiens forkhead box Q1 (Foxq1) was confirmed as a novel direct target gene of miR-124 by the dual-luciferase assay and western bolt.

Results: We found that miR-124 was commonly down-regulated in NPC specimens and NPC cell lines. The expression of miR-124 was inversely correlation with clinical stages and marked on T stages. Then, the ectopic expression of miR-124 dramatically inhibited cell proliferation, colony formation, migration and invasion in vitro, as well as tumor growth and metastasis in vivo. Furthermore, we identified Foxq1 as a novel direct target of miR-124. Functional studies showed that knockdown of Foxq1 inhibited cell growth, migration and invasion, whereas Foxq1 overexpression partially rescued the suppressive effect of miR-124 in NPC. In clinical specimens, Foxq1 was commonly up-regulated in NPC, and the level increased with clinical stages and T stages. Additionally, the level of Foxq1 was inversely correlated with miR-124.

Conclusions: Our results demonstrate that miR-124 functions as a tumor-suppressive microRNA in NPC, and that its suppressive effects are mediated chiefly by repressing Foxq1 expression. MiR-124 could serve as an independent biomarker to identify patients with different clinical characteristics. Therefore, our findings provide valuable clues toward the understanding the of mechanisms of NPC pathogenesis and provide an opportunity to develop new effective clinical therapies in the future.

Keywords: MicroRNA-124, Tumor growth, Metastasis, Nasopharyngeal carcinoma, Foxq1

\section{Background}

Nasopharyngeal carcinoma (NPC) is a non-lymphomatous squamous cell carcinoma derived from epithelial cells lining on the nasopharynx [1]. The characteristics are highly malignant local invasion and early distant metastasis, and the prevalence of NPC is low globally but high in southern China and Southeast Asia [2,3]. The radiotherapy proves to be the most sensitive and effective treatment, but the

\footnotetext{
* Correspondence: li321162@qq.com

${ }^{\dagger}$ Equal contributors

'Department of Otorhinolaryngology-Head and Neck Surgery, Nanfang Hospital, Southern Medical University, Guangzhou, Guangdong 510515, China

Full list of author information is available at the end of the article
}

average 5year survival rate for NPC patients remains at $70 \%$ [1,3-5]. During tumorigenesis and progression, multiple genetic and epigenetic abnormalities synergistically disrupt normal cell function, thus contributing to NPC pathogenesis [6-9]. Until now, the underlying mechanisms of NPC tumorigenesis are not completely understood. Therefore, further investigation is urgently needed.

MicroRNAs (miRs) are small non-coding RNAs which function as endogenous regulatory RNA molecules and modulate many physiological and pathological processes through down-regulating target genes $[10,11]$. There is a large body of evidence indicating that abnormal miRNAs 
can function as oncogenes or tumor suppressors in tumor progression [12]. To date, multiple miRNAs have been shown to be dysregulated in NPC, such as miR26a, miR-9, miR-10b, miR-144 and miR-214 which contributed to the development and progression of NPC [13-20]. Our previous study demonstrated for the first time that plasma miR-124 was down-regulated in NPC [21]. However, the role of miR-124 in NPC and the molecular mechanisms in which miR-124 exerts its functions remain largely unknown.

Human Foxq1 is first identified to encode a protein of 403 amino acids and belongs to the family of the Fox transcription factors (previously called HNF-3/ forkhead transcription factors) in 2001 [22]. The biological function of Foxq1 has been clearly identified in hair follicle differentiation [23,24]. Previous studies have found that Foxq1 is widely expressed at the mRNA level in murine tissues, with particularly high expression levels in the bladder and stomach [25]. Recent several studies reported that Foxq1 was markedly overexpressed in colorectal cancer and glioma, which enhanced tumorigenicity and tumor growth in vivo [26,27]. Furthermore, Foxq1 was also involved in epithelial-mesenchymal transition regulation by suppressing E-cadherin transcription, and associated with aggressive cancer phenotype [14,15]. However, whether Foxq1 expression contributes to NPC development and progression is not clear.

In this study, we investigated the potential involvement of miR-124 in NPC. We examined the expression level of miR-124 in human NPC cells and tissues and tested its effects on cell growth, migration and invasion. In addition, we also investigated a potential role of miR-124 on NPC tumorigenesis and tumor metastasis in murine models. Finally, we explored the underlying mechanism of miR-124 functions in NPC. Our study will provide a better understanding of NPC pathogenesis.

\section{Results}

MiR-124 was down-regulated in NPC cell lines and clinical specimens and associated with advanced clinical stage

To study the expression level of miR-124 in NPC, a panel of NPC cell lines was first analyzed by Real-time PCR. Compared with the immortalized nasopharyngeal epithelial cell line NP69, the basal expression level of miR-124 was generally down regulated in 7 NPC cell lines (5-8 F, 6-10B, CNE1, CNE2, HONE-1, C666-1 and Sune-1) (Figure 1A).

We further tested the expression level of miR-124 in 178 primary NPC tissues and 55 non-cancer nasopharyngitis biopsy samples to analyze the clinicopathologic significance of miR-124. The relationship between the miR-124 expression level and clinicopathologic characteristics in NPC patients were summarized in Table 1. MiR-124 was not significantly associated with age and gender of the patients. Consistent with the result of the NPC cell lines, the average expression level of miR-124 was decreased in NPC tissue compared with non-cancer biopsy samples (Figure 1B). We found that miR-124 expression was higher in stage I, whereas stages II-IV had lower levels, showing a significant correlation of miR124 with clinical stages (Figure 1C). Furthermore, we found that the miR-124 expression was higher in stage T1, whereas stages T2-T4 had lower levels, showing a significant correlation of miR-124 with T stage (Figure 1D). In addition, we found that the level of miR-124 was lower in distance metastasis tissues compared with local metastasis tissues (Figure 1E). Taken together, these data provided strong evidence that miR-124 expression was closely related to the progression and clinicopathologic features of NPC. On the basis of these results, we focused on miR-124 for further functional studies to evaluate its roles in NPC pathogenesis.

\section{MiR-124 suppressed the proliferative, migratory and invasive capacities of NPC cells}

To determine the role of miR-124, we transfected the NPC cell lines 5-8 F and 6-10B with miR-124 mimics and miR-Ctrl. The up-regulated expression of miR-124 was confirmed by Real-time PCR (Figure 2A). CCK-8 array was utilized to evaluate cell proliferative capacity. These results showed that ectopic expression of miR-124 could dramatically repress the cell growth of 5-8 $\mathrm{F}$ and $6-10 \mathrm{~B}$ cells respectively (Figure $2 \mathrm{~B}$ and $\mathrm{C}$ ). Then, we tested the cell cycle distribution. The results demonstrated that ectopic expression of miR-124 arrest G0 + G1 phase and decreased $S$ phase in 5-8 F and 6-10B cells compared with miR-Ctrl (Figure 3A). After analyzing the proliferation capacity of miR-124, we investigated the cell migration and invasion capacities. Migration and invasion arrays demonstrated that miR-124 overexpression inhibited cell migration and invasion compared with miR-Ctrl (Figure 2E, F, G and H).

To further investigate colony formation capacity, we used lentiviral vectors to stably up-regulate the expression level of miR-124 in 5-8 F and 6-10B cell lines (Figure 3B). Colony formation assay was performed to evaluate cell growth. Similar results indicated that miR-124 inhibited the colony formation ability in lv-miR-124/5-8 F and lvmiR-124/6-10B cells compared with lv-miR-Ctrl cells respectively (Figure $3 \mathrm{C}$ and $\mathrm{D}$ ). Above all, these results supported that ectopic expression of miR-124 inhibited cell growth, colony formation, migration and invasion in NPC cell lines in vitro.

\section{MiR-124 inhibited tumor growth and metastasis in vivo}

To determine whether miR-124 could affect tumor growth in vivo. Lv-miR-124/5-8 F cells and lv-miR-Ctrl cells were used for evaluating the effect of miR-124 overexpression on 


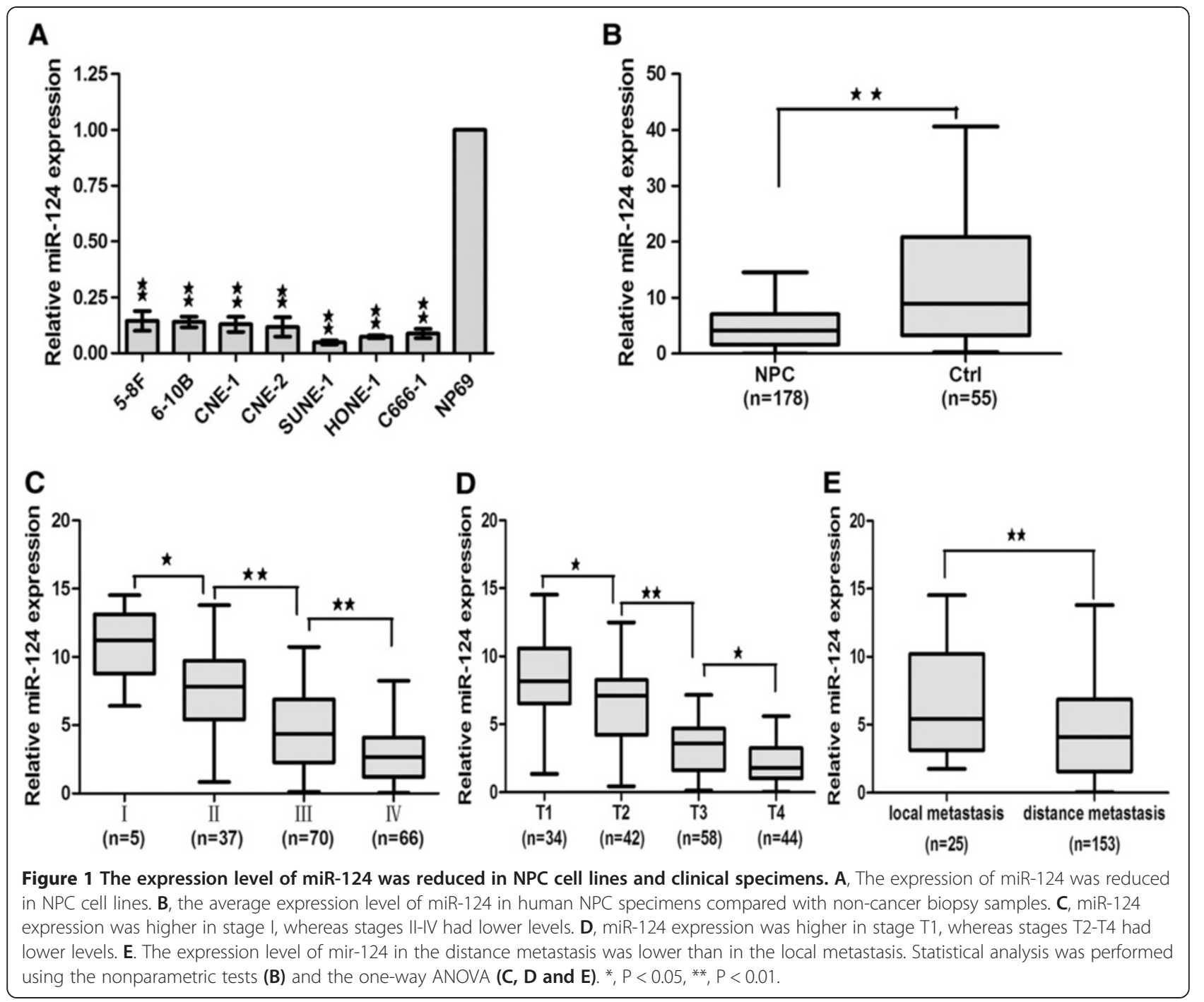

the growth of tumor xenografts. We implanted lv-miR-124/ 5-8 $\mathrm{F}$ or lv-miR-Ctrl/5-8 F cells subcutaneously in nude mice respectively ( $\mathrm{n}=6$ per group). As shown in Figure $3 \mathrm{E}$, lv-miR-124/5-8 F cells resulted in an approximately 2.68fold decrease in tumor size relative to lv-miR-Ctrl/5-8 F cells after 15 days (Figure 3F, $P<0.05$ ). After the tumors were dissected and weighed, the results of tumor weight were similar to results of tumor volume (Figure $3 G$ and $H$ ). The expression levels of miR-124 were examined after the tumors were dissected (Additional file 1: Figure S1A). Thus, these results showed that miR-124 suppressed tumor proliferation capacity in vivo. We also showed that both the staining intensity and the number of hyperproliferative Ki$67^{+}$and $\mathrm{PCNA}^{+}$tumor cells were significantly decreased compared with control (Figure 3I and Additional file 2: Table S1; $\mathrm{P}<0.05$ ).

To further investigate the metastatic effect of miR-124 in vivo, primary tumors were established by direct injection of lv-miR-124/5-8 F or lv-miR-Ctrl/5-8 F cells into the liver. Twenty-five days after transplantation, the mice were killed and the livers and lungs were dissected for macroscopic and microscopic histology. Livers and lungs of mice bearing miR-124-expressing 5-8 F tumors harbored statistically significantly fewer microscopic and macroscopic metastases than those of mice bearing mock-infected 5-8 F tumors $(P<0.05$; Figure 4A-D). The expression levels of miR-124 were examined after the tumors were dissected from the livers (Additional file 1: Figure S1B).

\section{MiR-124 down-regulated the expression of Foxq1 by directly targeting its 3 '-UTR}

To investigate the molecular mechanism for the proliferation, migration and invasion of suppression by miR124 in NPC cells, we focused on 27 possible target genes of mir-124 by utilizing bioinformatic analysis. The selected target genes have been verified that could affect tumor growth or metastasis in the website 
Table 1 The relationship between miR-124 expression and clinicopathological parameters in 178 NPC patients

\begin{tabular}{|c|c|c|c|c|}
\hline \multirow[t]{2}{*}{ Variable } & \multirow[t]{2}{*}{ No. } & \multicolumn{2}{|c|}{ Median expression of miR-124 } & \multirow[t]{2}{*}{$P$} \\
\hline & & High expression & Low expression & \\
\hline Age, year & & & & 0.188 \\
\hline$<45$ & 95 & $46(48.4 \%)$ & $49(51.6 \%)$ & \\
\hline$\geq 45$ & 83 & $43(51.8 \%)$ & $40(49.2 \%)$ & \\
\hline Gender & & & & 0.749 \\
\hline Male & 120 & $57(47.5 \%)$ & $63(52.5 \%)$ & \\
\hline Female & 58 & $32(55.1 \%)$ & $26(44.9 \%)$ & \\
\hline T status & & & & $<0.001$ \\
\hline $\mathrm{T} 1$ & 34 & $34(100 \%)$ & $0(0 \%)$ & \\
\hline $\mathrm{T} 2$ & 42 & $33(78.6 \%)$ & $9(21.4 \%)$ & \\
\hline $\mathrm{T} 3$ & 58 & $18(31 \%)$ & $40(69 \%)$ & \\
\hline $\mathrm{T} 4$ & 44 & $6(13.6 \%)$ & $38(86.4 \%)$ & \\
\hline $\mathrm{N}$ status & & & & 0.148 \\
\hline NO & 16 & $10(62.5 \%)$ & $6(37.5 \%)$ & \\
\hline N1 & 80 & $44(55 \%)$ & $36(45 \%)$ & \\
\hline N2 & 60 & 27 (45\%) & 33 (55.0\%) & \\
\hline N3 & 22 & $8(36.4 \%)$ & $14(63.6 \%)$ & \\
\hline M status & & & & 0.114 \\
\hline MO & 166 & $82(50 \%)$ & $84(50 \%)$ & \\
\hline M1 & 12 & 7 (58.0\%) & $5(42.0 \%)$ & \\
\hline \multicolumn{5}{|l|}{ Stage } \\
\hline I & 5 & $5(100 \%)$ & $0(0 \%)$ & $<0.001$ \\
\hline$\|$ & 37 & 33 (89.1\%) & $4(10.8 \%)$ & \\
\hline III & 70 & $35(50 \%)$ & $35(50 \%)$ & \\
\hline IV & 60 & $16(26.7 \%)$ & $44(73.3 \%)$ & \\
\hline
\end{tabular}

(http://www.ncbi.nlm.nih.gov/nucleotide/). The selected targets were validated by RT-qPCR in lv-miR-124/5-8 F cells. The result of RT-qPCR showed that the expression level of Foxq1 decreased more significantly, compared with other 26 possible target genes (Additional file 3: Figure S2A). Finally, we selected Foxq1 gene as the target gene of miR-124. Foxq1 is highly conserved among different species, whose 3'-UTR of mRNA contained a complementary site for the seed region of miR-124 (Figure 5A). Real-time PCR and western blot was performed to detect the expression level of Foxq1, and the results showed that Foxq1 was up-regulated in 7 NPC cell lines compared with NP69 (Figure 5B and C). Then we analyzed the the mRNA and protein level of Foxq1 after transfected lv-miR-124 into 5-8 F and 6-10B cell lines. As shown in Figure 5D and Additional file 3: Figure S2B, Foxq1 was downregulated after transfected lv-miR-124.

To further confirm that Foxq1 was a direct target of miR-124, dual-luciferase assay was performed. The target region sequence of Foxq1 3'-UTR (wt 3'-UTR) or the mutant sequence (mut $3{ }^{\prime}$-UTR) was cloned into a luciferase reporter vector (Figure 5E). These recombinant vectors were co-transfected with miR-124 mimics or miR-Ctrl into 5-8 F cell line. The results showed that miR-124 could down-regulated the luciferase activity of the Foxq1 wt 3'-UTR construct (Figure 5F, lanes 1 and $2 ; \mathrm{P}<0.01$ ). The activity of mut3'-UTR vector was unaffected by a simultaneous transfection with miR-124 (Figure 5F, lanes 3 and 4). Moreover, miR-Ctrl did not significantly affect the luciferase activity of either the wt or mut 3'-UTR construct (Figure 5F, lanes 5 and 6). The same results were obtained in HEK $293 \mathrm{~T}$ cell line (Additional file 3: Figure S2C). In summary, these results strongly suggested that miR-124 directly regulated Foxq1 in NPC cell lines.

\section{Overexpression of Foxq1 could partially rescue the suppression of miR-124}

To explore the function of Foxq1 in NPC cell lines, Foxq1siRNAs were transfected into 5-8 F cell lines. Real-time PCR was confirmed that the Foxq1 mRNA level expression was down-regulated compared with miR-Ctrl after transfected (Additional file 4: Figure S3A). CCK8 array showed that Foxq1 silencing inhibited the proliferation (Additional file 4: Figure S3B). Furthermore, migration and invasion arrays obtained the same results that knockdown of Foxq1 repressed the migration and invasion capacities of 5-8 F cells (Additional file 4: Figure S3C and D). According to these results, Foxq1 functioned as a potential oncogene in NPC cell lines.

To elucidate whether the suppressive effect of miR124 was mediated by repression of Foxq1 in NPC cells, we performed gain-of-function and loss-of-function studies. First, we silenced Foxq1 to investigate whether the reduced expression of Foxq1 could mimic the suppressive effect of miR-124. 5-8 F cells were transfected with siRNA-Foxq1 or miR-124 mimics. The Foxq1 protein expression of siRNA-Foxq1/5-8F and miR-124/5-8F were down-regulated compare with siRNA-Ctrl/5-8F (Figure 6D). Then, we examined cell proliferative, migratory and invasive capacities. As shown in Figure 6A-C, Foxq1 knockdown led to significant suppressive effects, similar to those induced by miR-124 $(P<0.01)$. The similar results were obtained in 6-10B cell lines (Additional file 4: Figure S3E-G). Subsequently, we evaluated whether ectopic expression of Foxq1 could rescue the suppressive effect of miR-124. The lv-miR-124/5-8 F cell line after transfected lv-Foxq1 was named lv-miR-124 + lv-Foxq1/ 5-8 F. The expression level of Foxq1 was tested using Real-time PCR and Western bolt (Figure 6I and J). CCK8 array and colony-forming assay showed that Foxq1 could partially abrogate the effects mediated by miR-124 in lvmiR-124 + lv-Foxq1/5-8 F cells (Figure 6E and F). At the same time, the migration and invasion arrays showed 


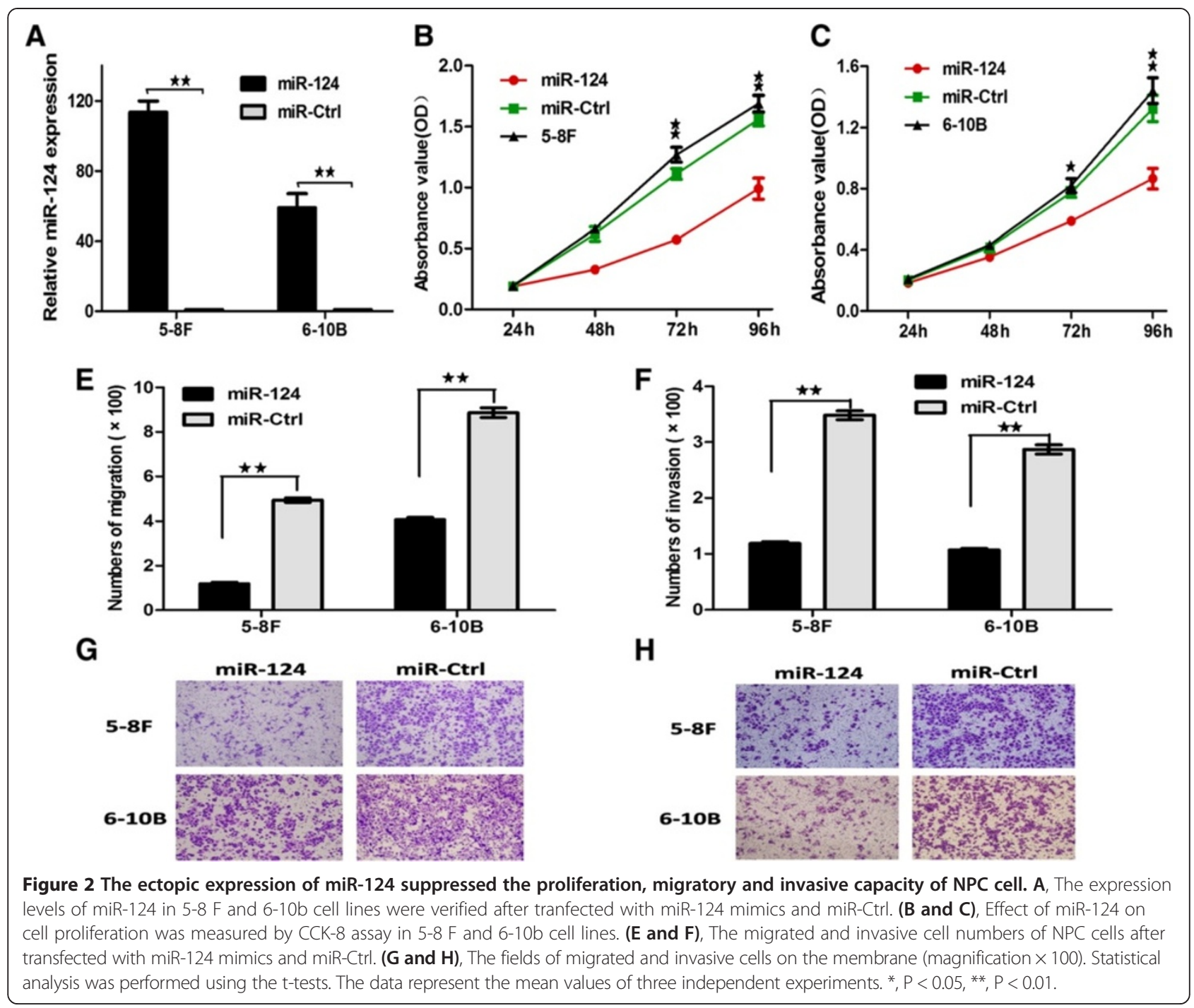

that Foxq1 could partially restore the migration and invasion activity compared with the lv-Ctrl/5-8 F cells (Figure $6 \mathrm{G}$ and $\mathrm{H}$ ). The similar results were obtained in lv-miR-124 + lv-Foxq1/6-10B cell lines (Additional file 5: Figure S4A-F). Therefore, we showed that Foxq1 could partially rescue the suppression of miR-124 in NPC cells.

\section{MiR-124 and Foxq1 were inversely correlated in NPC tissues}

To further investigate the expression of Foxq1, 178 clinical human primary NPC tissues and 55 non-cancer nasopharyngitis biopsy samples were tested for analyzing the clinicopathologic significance. We found that the average expression level of Foxq1 was up-regulated in NPC tissue compared with non-cancer biopsy samples (Figure 7A). Further analysis found that the Foxq1 expression of clinical I stage was the lowest in the clinical stages (Figure 7B). In addition, we found that Foxq1 expression was lower in stage $\mathrm{T} 1$, whereas stages $\mathrm{T} 2$ $\mathrm{T} 4$ had higher levels, showing a significant correlation of Foxq1 with T stage (Figure 7C). We then correlated Foxq1 with the miR-124 expression levels in the same NPC specimens. As shown in Figure 7E, significant inverse correlation was observed when Foxq1 expression levels were plotted against miR-124 expression levels (2-tailed Spearman's correlation, $\mathrm{r}=-0.5646 ; p<0.001)$.

Immunohistochemistry (IHC) was also performed to detect Foxq1 expression in 178 clinical NPC specimens and 55 non-cancer nasopharyngitis biopsy samples. Foxq1 was found to be predominantly overexpressed in the cytoplasm and membranes of NPC cells and was less expressed in non-cancer nasopharyngitis biopsy samples (Figure 7D). Our data also showed that the Foxq1 expression was statistically higher than in non-cancer biopsy samples. We found that Foxq1 expression was lower in stage I, whereas stages II-IV had higher levels, showing a significant correlation of Foxq1 with clinical 


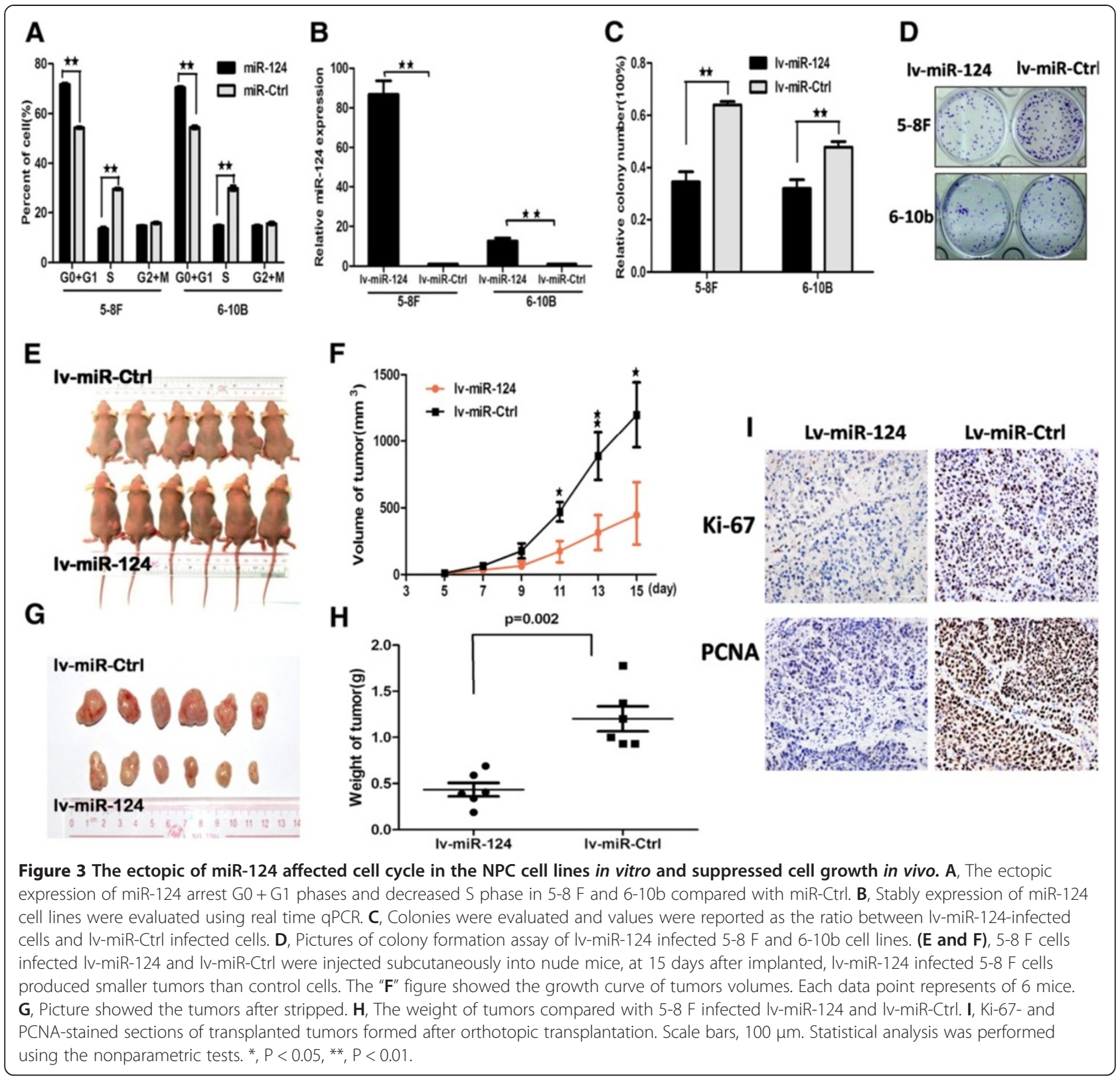

stages $(\mathrm{P}<0.05$, Figure $7 \mathrm{~F})$. Then we correlated Foxq1 with the miR-124 expression levels in the same NPC specimens. As shown in Figure 7G, a significant inverse correlation was observed when Foxq1 expression levels were plotted against miR-124 expression levels (2-tailed Spearman's correlation, $\mathrm{r}=-0.6056 ; p<0.0001)$.

\section{Discussion}

In this report, we identified for the first time that miR124 was markedly down-regulated in NPC cell lines and clinical specimens. The ectopic expression of miR-124 suppressed the proliferative, migratory and invasive capacities of NPC cells in vitro, and suppressed tumor growth and metastasis in vivo. Moreover, we found that the expression of Foxq1 protein was down-regulated after transfected lv-miR-124 in NPC cells by western blot. By using Dual-luciferase assay, Foxq1 was identified as a new direct and functional target of miR-124. Our results also showed that Foxq1 overexpression could rescue partially the suppressive effect of miR-124, and we found a negative correlation between miR-124 and Foxq1 expression in NPC tissues. These results supported that Foxq1 was a direct target gene of miR-124. Our study demonstrated that miR-124 acts as a novel proliferation and metastasis suppressor by targeting Foxq1 in NPC.

The ectopic expression of miR-124 is a frequently epigenetically silenced tumor-suppressive microRNA in various cancers [28-34]. The capability of cells to proliferate, 


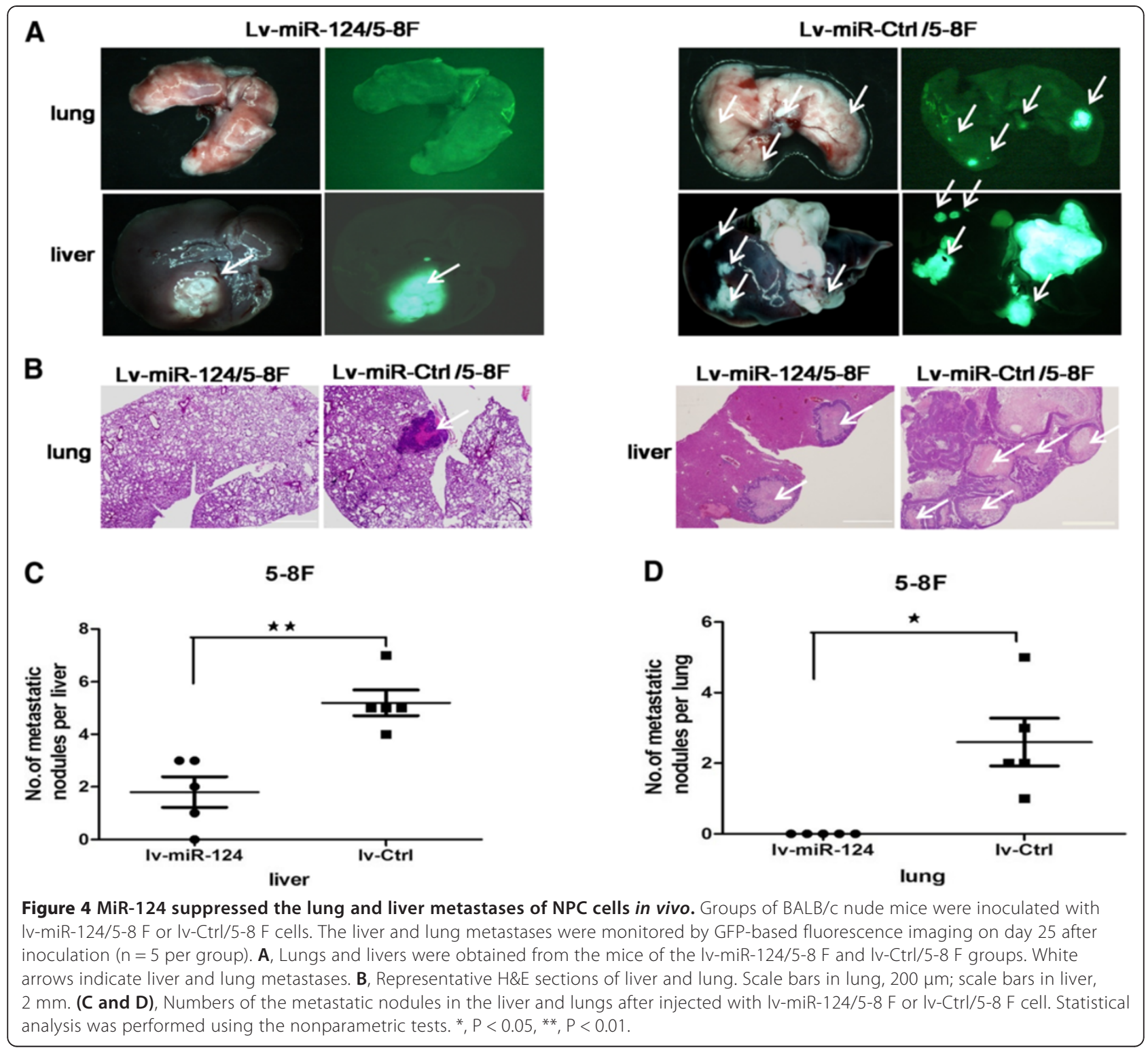

migrate and invade is considered an important determinant in the process of tumorigenesis and progression. Many oncogenes and suppressor genes reportedly correlate with the course of cancer initiation and progression [28]. In this study, our results provided strong evidences that miR-124 inhibited the proliferation, migration and invasion of NPC cells in vitro and in vivo. These results were consistent with observations in breast cancer, hepatocellular carcinoma and glioblastoma [28-34]. The suppressive capability suggested that miR-124 functioned as a tumor-suppressive microRNA in NPC.

Emerging evidence indicates that miR-124 is abnormally expressed and has been implicated in several tumors. Until now, several studies have demonstrated that miR-124 was down-regulated and inversely correlated with clinical characteristic and prognosis in breast cancer and colorectal cancer [28,35]. Our previous study demonstrated that plasma miR-124 was down-regulated in NPC. The present study is the first report to explore the expression of miR-124 in NPC tissues. We found that the expression level of miR-124 between NPC patient plasma and tissue decreased simultaneously. The reduced expression of miR-124 in NPC tissues was inversely correlated with clinical stages, $\mathrm{T}$ stages and marked the progression from locoregional tumors to metastatic tumors. Recent reports have showed that miRNAs which can be reliably isolated from human plasma are stable in circulation, and can be used as a diagnostic tool for early detection of NPC $[11,36]$. More importantly, it is simple, effective, and non-invasive blood-based biomarker which predicts the clinical behaviour of NPC and monitored therapeutic response 


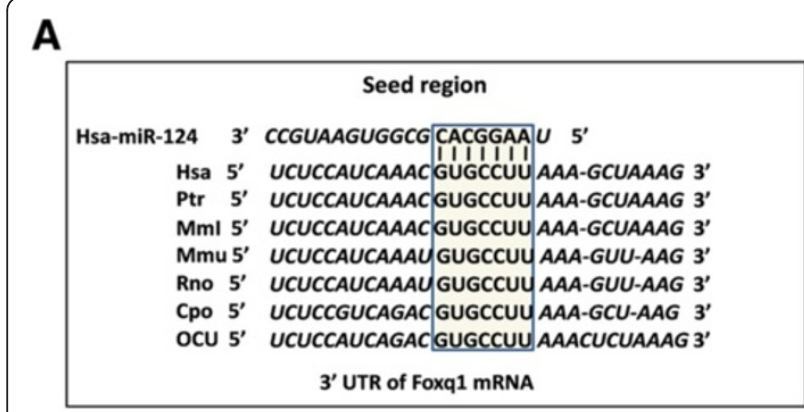

C

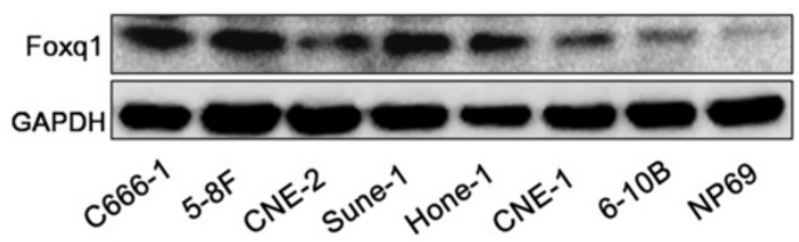

$\mathbf{E}$

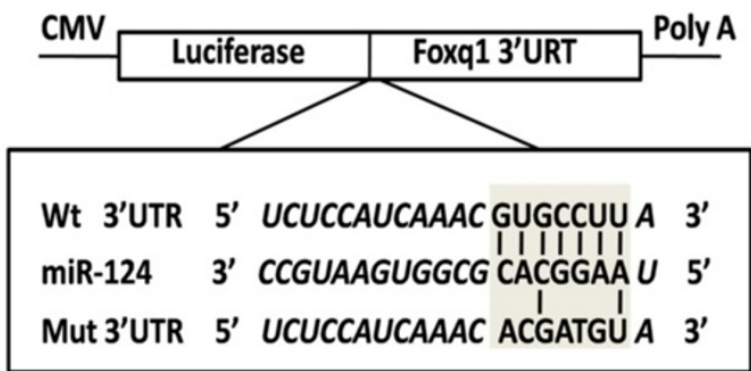

B

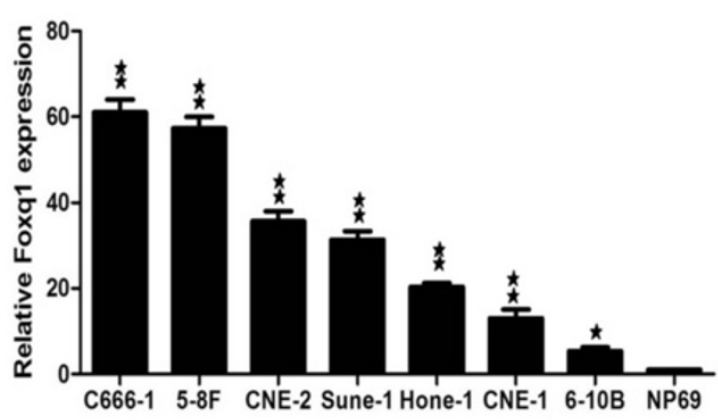

D
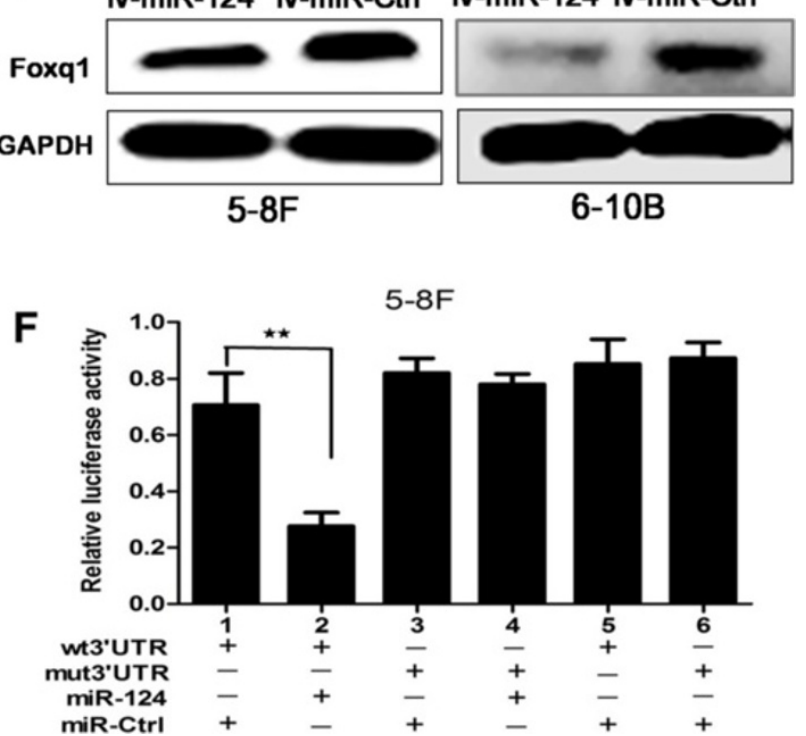

Figure 5 The ectopic miR-124 induced the expression of Foxq1 by directly targeting its 3'-UTR. A, Putative miR-124 binding site in the $3^{\prime}-$ UTR region of Foxq1 and interspecies conservation of seed matching sequences (gray box). (B and C), The gene expression of Foxq1 in NPC cells compared with NP69 cells by RT-qPCR and western bolt. D, The protein expression level of Foxq1 in IV-miR-124/5-8 F cell and IV-miR-124/6-10B cell compared with control. E, Diagram of wt 3'-UTR and mut 3'-UTR of Foxq1 contained reporter constructs. F, Luciferase reporter assays in 5-8 F cells, co-transfected of wt/mut 3'-UTR with miRNAs as indicated. Statistical analysis was performed using the t-tests. The data represent the mean values of three independent experiments. ${ }^{*}, \mathrm{P}<0.05,{ }^{*}, \mathrm{P}<0.01$.

[21]. Compared with the results in the present study, the expression level of miR-124 between NPC patient plasma and tissue changed in the same direction. The reduced expression of miR-124 in NPC tissues was inversely correlated with clinical characteristic. For these reasons, miR-124 could serve as an independent biomarker marker to identify patients with clinical characteristic. However, further investigation is needed to confirm the prognostic value of miR-124 as an effective biomarker.

Foxq1 is a member of the FOX gene family. FOX genes are involved in embryonic development, cell cycle regulation, tissue-specific gene expression, cell signaling, and tumorigenesis [22,37]. Recent studies have clearly showed that Foxq1 was implicated in tumor proliferation and metastasis in colorectal cancer, glioblastoma, breast cancer and hepatocellular carcinoma [26,27,38,39]. Foxq1 was a prognostic marker for patients in gastric cancer and hepatocellular carcinoma $[40,41]$. However, the role of Foxq1 is rarely known in NPC. Our results demonstrated for the first time that Foxq1 was overexpressed in NPC cell lines and NPC tissues, consistent with the published data $[27,39,40,42]$. In NPC tissues, Foxq1 expression increased with clinical stage, $\mathrm{T}$ stage. Functional studies also confirmed that down-regulation of Foxq1 suppressed cell proliferation, migration and invasion in vitro. These results implied that Foxq1 was a potential oncogene in development and progression of NPC. Nonetheless, the molecular pathological mechanism of Foxq1 in NPC was still unknown. A report showed that Foxq1 may be a novel target of the Wnt pathway in solid tumors and Foxq1 expression was associated with genes as markers for proliferation (MKI67, TPX2, AURKA) and epithelial-mesenchymal transition (VIM, SNAI1, ZEB2, CDH2) [42]. Then, we tested 


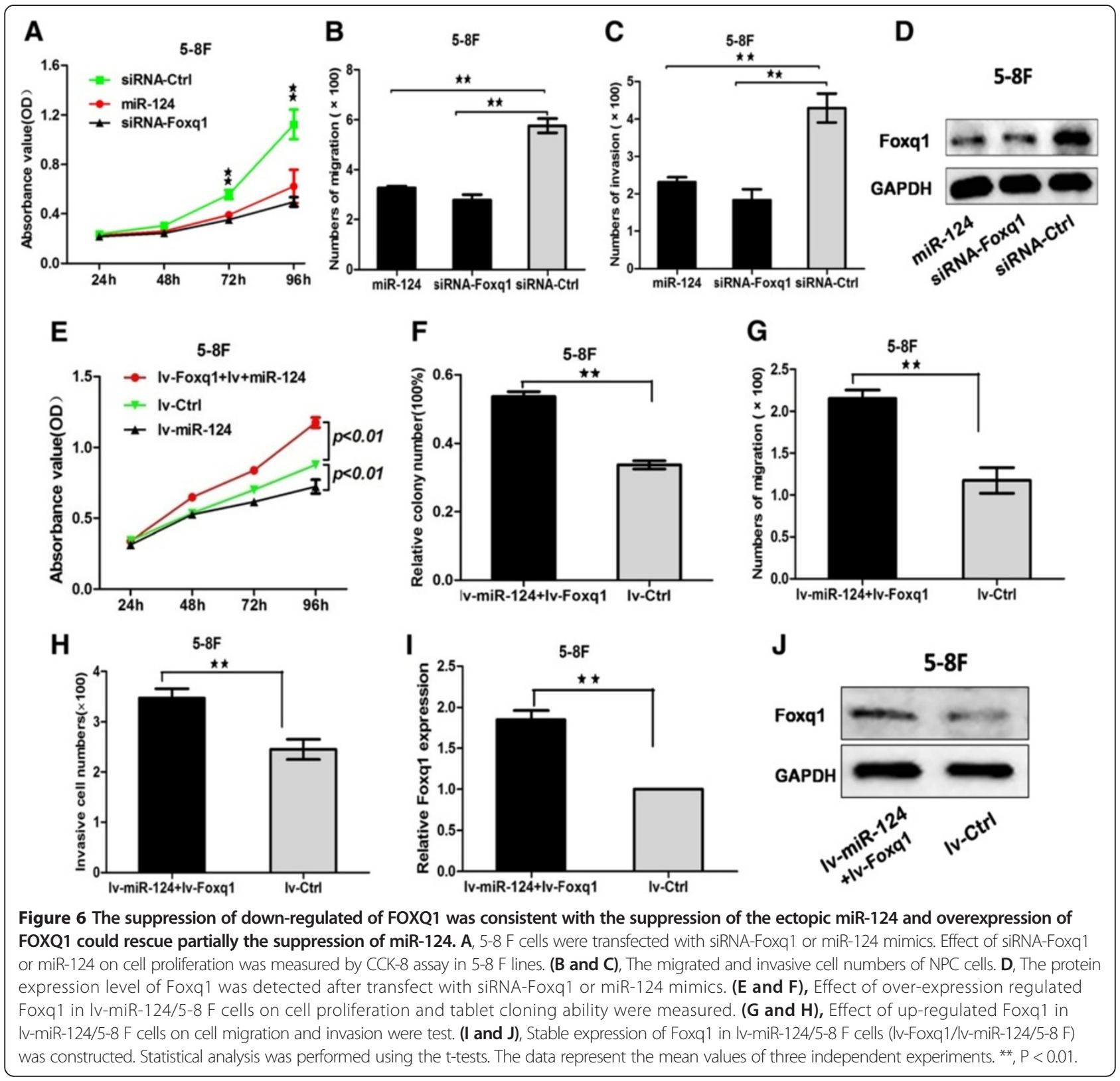

the mRNA levels of these genes and found that these genes (MKI67, SNAI1 and ZEB2) were down-regulated after siRNA- Foxq1 transfection in NPC cells (data not shown). Further study is needed to clearly illustrate the tumorigenic mechanisms of Foxq1.

\section{Conclusions}

This study identifies miR-124 as a growth suppressive miRNA in human NPC, at least, partly through repression of Foxq1. MiR-124 could serve as an independent biomarker marker to identify patients with clinical characteristic. Although miRNA-based therapeutics is still in their infancy, our findings on miR-124 are encouraging and suggest that this miRNA could be a potential target for the treatment of NPC in future.

\section{Methods}

\section{Clinical specimens}

Tumors biopsy specimens $(\mathrm{n}=178)$ and non-cancer nasopharyngitis biopsy samples $(n=55)$ were obtained from patients in Nanfang Hospital (Southern Medical University, Guangzhou, China) and were frozen in liquid nitrogen for further study. Informed written consent was obtained from each patient. All samples were pathologically confirmed as NPC. The TNM classification was according to the definitions of the seventh edition of the UICC-American Joint Committee on Cancer staging 

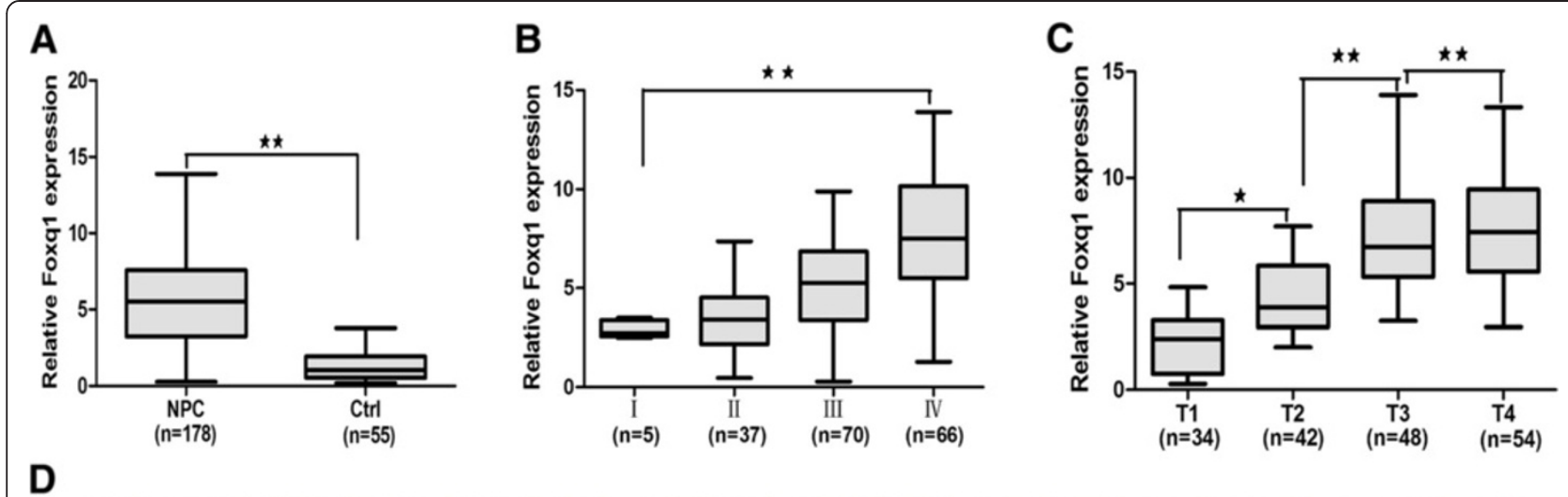

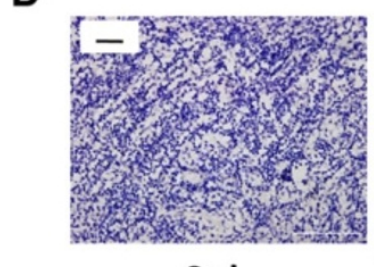

Ctrl

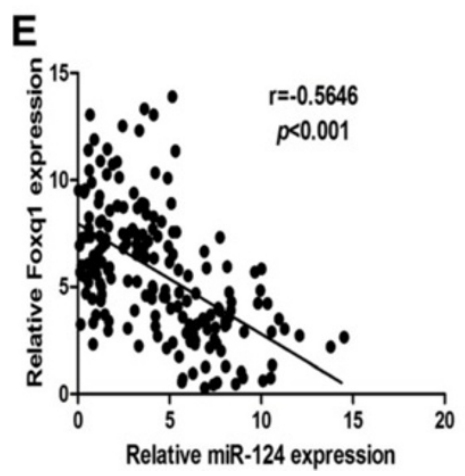

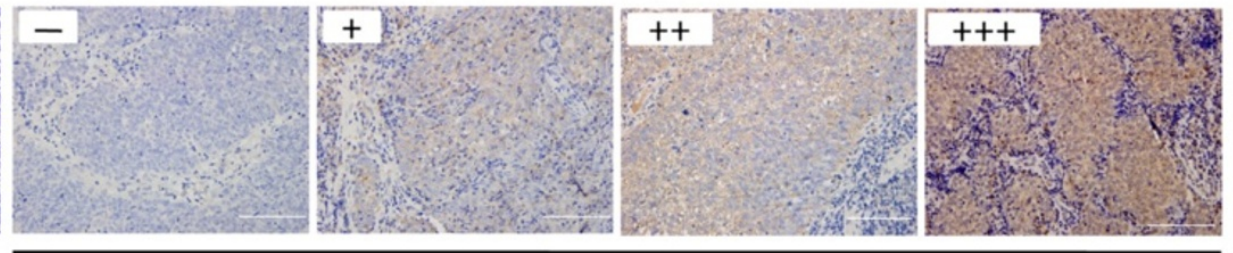

NPC

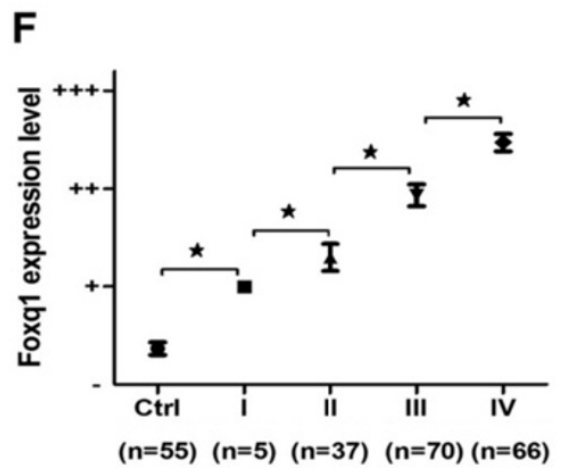

G

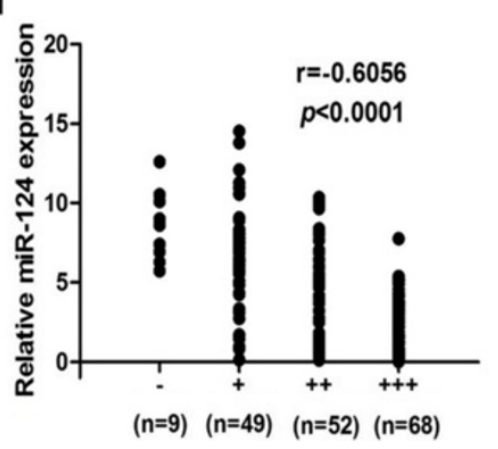

Figure 7 Mir-124 and Foxq1 are inversely correlated in NPC tissues. A, The average expression level of Foxq1 in human NPC specimens compared with non-cancer biopsy samples. B, The Foxq1 expression of clinicallstage had lower expression than in the IVstage. C, Foxq1 expression was lower in stage T1, whereas stages T2-T4 had higher levels. D, Representative IHC for Foxq1 in non-cancer biopsy samples and NPC specimen with different clinical stages. E, In the mRNA levels, a significant inverse correlation was observed after correlated Foxq1 with the miR-124 expression levels in the 178 NPC specimens. F, Statistical quantification of the IHC scores of Foxq1 between non-cancer biopsy samples and NPC specimen with different clinical stages Scale bars, $100 \mu \mathrm{m}$. G, A scatter diagram shows an inverse correlation between miR-124 and Foxq1 expression in the same set of NPC tissue (Spearman's correlation analysis, $r=-0.6056 ; p<0.0001)$.Statistical analysis was performed using the nonparametric tests (A) and the one-way ANOVA $(B, C, E, F) . *{ }^{*}, P<0.05, * *, P<0.01$.

criteria. The research protocols were approved by the Ethics Committee of Nanfang Hospital and registered in Clinical.trials.gov (ID: NCT01171235).

\section{Cell culture}

Human nasopharyngeal carcinoma cell lines such as 5-8 F, 6-10B, CNE1, CNE2, Sune-1, Hone-1 and C6661 were cultured in RPMI-1640 medium (HyClone, Thermo scientific Inc, China). HEK $293 \mathrm{~T}$ cell line (Cell Bank of Chinese Academy of Science in Shanghai, China) was cultured in DMEM/High Glucose medium (HyClone, Thermo scientific Inc, China). The above cell lines were supplemented with $10 \%$ fetal bovine serum (FBS, HyClone, Thermo scientific Inc, China).
The immortalized nasopharyngeal epithelial cell NP69 (Cancer Research Center, Southern medical university, China) was cultured as the control cell line in Keratinocyte-SFM (GIBICO, Life Technologies corporation, USA).

\section{RNA isolation, reverse transcription, and quantitative Real-time PCR}

Total RNA was extracted from the samples using RNAiso Plus (TAKARA, Shiga, Japan) and reversely transcribed to cDNA using the All-in-One First-Strand cDNA Synthesis kit (GeneCopoeia Inc., USA). Quantitative Real-time PCR (qPCR) was performed using All-in$\mathrm{One}^{\mathrm{TM}}$ qPCR Mix (Applied GeneCopoeia Inc., USA) on 
an ABI 7500HT System. U6 and GAPDH snRNA were used as miRs and miRNA endogenous control respectively. All samples were normalized to the internal control, and the relative expression levels of miR-124 and Foxq1 were calculated using relative quantification assay. Primer sequences for qRT-PCR were displayed in Additional file 6: Table S2.

\section{Oligo-nucleotide transfection}

MiR-124 mimics, miR-Ctrl, Foxq1 siRNAs and Foxq1Ctrl were synthesized from Gene-pharma (Shanghai, China). RNA oligonucleotides were transfected by lipofectamine 2000 reagent (Invitrogen, Carlsbad, CA, USA).

\section{Plasmid construction and stable transfection}

To obtain stable cell lines to overexpress miR-124, pre-miR-124 was cloned into the pLVTHM lentiviral vector, and the recombinant plasmid was named as lv-miR-124. The lentivial vectors and packing system (psPAX2 and PMD2G) were co-transfected in HEK293T cells by calium phosphate precipitation. Then lentivial virus was collected to infect the 5-8 F and $6-10 \mathrm{~B}$ cell lines.

The 3'-UTR of Foxq1 was amplified from human genomic DNA and cloned into the psicheck.2 vector (Promega, USA). The mutation of the 3'-UTR of Foxq1 was carried out using site-directed mutagenesis kit (Invitrogen, Carlsbad, CA, USA).

The lentivial viruses to overexpress Foxq1 were purchased from Genechem (Shanghai, China). Then they were used to infect 5-8 F cell lines which stably overexpressed miR-124. The transfected cells were selected by Flow Cytometry.

\section{Bioinformatics analysis}

The probable target genes of miR-124 were predicted using three microRNA target database (PicTar, TargetScan and PITA), and the selected targets gene were validated by RT-qPCR and Western Blot.

\section{Dual-luciferase assay}

Cells were cultured in 24-well plates for Dual-luciferase report system. Cells were co-transfected with $200 \mathrm{ng}$ Wild or Mutant type reporter plasmid and $20 \mathrm{nmol}$ miRNA or anti-miRNA using lipofectamine 2000 reagent (Invitrogen). Cells were harvested and lysed after 24 hours transfection. The firefly and Renilla luciferase activities were measured using the Dual-Glo luciferase reporter assay kit (Promega, Madison, WI, USA).

\section{Cell proliferation and colony-formation assay}

To determine the effect of miR-124 on cell proliferation, cells with stable overexpression of miR-124 were seeded into 96 wells plates at a density of $1 \times 10^{3}$ cells/well with five replicate wells. The effect of miR-124 on cell growth and viability was determined by Cell counting kit 8 assay as described previously [43]. To measure colony-forming activity, cells were counted and seeded into 12-well plates at 100 cells per well. Twelve days after seeding, the numbers of colonies containing more than 50 cells were stained with crystal violet and counted.

\section{Migration and invasion assays in vitro}

Transwell migration assay and invasion assay were conducted to determine the functional effects of miR-124 on cell migration and invasion as described previously [14].

\section{Cell cycle assays}

To determine cell cycle distribution, the cells were plated in 6-well plates and transfected with miRNA mimics or siRNA duplexes. After transfection, the cells were harvested, treated with cell cycle detection kit (keygentec Nanjing China) and tested using a FACSCalibur instrument (Becton Dickinson, CA, USA). The data were analyzed using the CellQuest Pro software (BD Biosciences).

\section{Tumor xenograft model and tumor metastasis assay in vivo}

Twelve female BALB/C nude mice about four to fiveweek-old were purchased from Centre of Laboratory Animal of Southern Medical University, and the animal protocol was approved by Institutional Animal Care and Use Committee of Southern Medical University. Twelve nude mice were randomly divided into two groups. $1 \times$ $10^{5} \mathrm{lv}$-miR-124/5-8 $\mathrm{F}$ or lv-miR-Ctrl/5-8 $\mathrm{F}$ cells were injected into the dorsal flank of each mouse. Tumor size was measured every other day. Mice were sacrificed and tumors were dissected and weighed. Tumor volumes were calculated as follows: volume $=\left(\mathrm{D} \times \mathrm{d}^{2}\right) / 2$, where $\mathrm{D}=$ the longest diameter and $\mathrm{d}=$ the shortest diameter.

For tumor metastasis assay in vivo, we used a murine model of NPC metastasis successfully established previously [44]. To establish this model, we inoculated NPC cells into the liver as a single nodule, which would subsequently metastasized to other parts of the liver and the lung. Ten nude mice were randomly divided into two groups. $1 \times 10^{6} \mathrm{lv}-\mathrm{miR}-124 / 5-8 \mathrm{~F}$ or lv-miR-Ctrl/5-8 F cells were injected into the liver of each mouse. The mice were killed and autopsied on day 25 , and the incidence of lung or liver metastasis were recorded.

\section{Western blot}

Protein lysates extracted from cell lines were separated by $10 \%$ SDS-PAGE, and electrophoretically transferred to PVDF (polyvinylidene difluoride) membrane (Millipore). Then, the membrane was incubated with goat polyclonal antibody against human Foxq1 (WuXi PharmaTech Cayman China) followed by HRP (horseradish peroxidase)- 
labeled goat-anti mouse IgG (Santa Cruz Biotechnology) and detected by chemiluminescence. Glyceraldehyde-3phosphate dehydrogenase (GAPDH) was used as a proteinloading control.

\section{Immunohistochemical analysis}

Formalin-fixed, paraffin-embedded tissues of transplanted tumors were sectioned at 4-mm thickness and analyzed for anti-Foxq1 primary antibody (Abcam Ltd, Cambridge, UK), anti- Ki-67 primary antibody, and antiPCNA primary antibody (Cell Signaling Technology, Inc. USA). Visualization was achieved using the EnVisionp peroxidase system (Dako). A sample was considered positive if more than $50 \%$ of the tumor cells retained nuclear staining, and 5 fields were randomly selected according to semiquantitative scales. The intensity of staining was scored manually: negative $(-)$, weak positive $(+)$, medium positive $(++)$, strong positive $(+++)$. Data recording and analysis by 2 independent experienced pathologists, and only tumor cells were scored.

\section{Statistical analysis}

Statistical analyses were conducted using spss13.0 statistical software. All experiments were performed for three times. The data are shown as the mean \pm SEM unless otherwise noted. Two-tailed Student's $t$ test was used for comparison of two independent groups. A one-way ANOVA analysis of variance was used to compare multiple groups. MiR-124 and Foxq1 expressions between tumor and control specimens were analyzed by a MannWhitney $U$ test. The relationship between miR-124 and Foxq1 was analyzed using Spearman's correlation analysis. $P$ values of $<0.05$ were considered statistically significant.

\section{Additional files}

Additional file 1: Figure S1. The expression levels of miR-124 in tumor xenograft model and tumor metastasis assay in vivo. $\mathbf{A}$, The expression levels of Lv-miR-124/5-8F compared with Lv-miR-Ctrl/5-8F in tumorigenesis in murine models. B. The expression levels of Lv-miR-124/5-8F compared with Lv-miR-Ctrl/5-8F in tumor metastasis murine models. Statistical analysis was performed using the t-tests. The data represent the mean values of three independent experiments. **, $\mathrm{P}<0.01$

Additional file 2: Table S1. Immunohistochemical analysis of Ki-67 and 662 PCNA expression in transplanted tumors of nude mice.

Additional file 3: Figure S2. The ectopic miR-124 induced the expression of Foxq1 by directly targeting its $3^{\prime}-\mathrm{UTR}$. A , The 27 possible target genes of mir-124 were predicted by bioinformatic analysis. Results of qRT-PCR showed the relative expression of Foxq1 was the most significant down-regulated in Iv-miR-124/5-8F cells compared with Iv-miR-Ctrl/5-8F. B, The mRNA expression level of Foxq1 in Iv-miR-124/5-8F cell and Iv-miR-124/6-10B cell compared with control cells. C, Luciferase reporter assays in HK293T cells, co-transfected of wt/mut 3'-UTR with miRNAs as indicated. Statistical analysis was performed using the t-tests. The data represent the mean values of three independent experiments.

* $P<0.05$, **, $P<0.01$

Additional file 4: Figure S3. Down-regulated of Foxq1 inhibited cell proliferation, migration and invasion and the suppression of down-regulated of Foxq1 was consistent with the suppression of the ectopic miR-124. A, The mRNA expression levels of Foxq1 in 5-8F cells after tranfected with Foxq 1 siRNA and Ctrl-siRNA. B, Effect of down-regulated Foxq1 on cell proliferation was measured by CCK-8 assay used 5-8F cell lines. (C and D), The migratory and invasive cell numbers were detected after tranfected with Foxq1 siRNA and Ctrl-siRNA. E, 6-10B cells were transfected with siRNA-Foxq1 or miR-124 mimics. Effect of siRNA-Foxq1 or miR-124 on cell proliferation was measured by CCK-8 assay in 6-10B lines. ( $\mathbf{F}$ and $\mathbf{G}$ ), The migrated and invasive cell numbers of $6-10 B$ cells. Statistical analysis was performed using the t-tests. The data represent the mean values of three independent experiments. ${ }^{*}, \mathrm{P}<0.05,{ }^{* *}, \mathrm{P}<0.01$.

Additional file 5: Figure S4. Over-expression of Foxq1 could rescue partially the suppression of miR-124 in 6-10B cells. (A and B), Effect of up-regulated Foxq1 in Iv-miR-124/5-8F cells (Iv-Foxq1/lv-miR-124/6-10B) on cell proliferation and tablet cloning ability were measured. (C and D), Effect of up-regulated Foxq1 in Iv-miR-124/5-8F cells on cell migration and invasion was test. (E and $\mathbf{F}$ ), Stable expression of Foxq1 in Iv-miR124/6-10B cells (Iv-Foxq1/lv-miR-124/6-10B) was constructed. Statistical analysis was performed using the t-tests. The data represent the mean values of three independent experiments. ${ }^{*}, \mathrm{P}<0.01$.

Additional file 6: Table S2. Primers was used in this study.

\section{Abbreviations}

NPC: Nasopharyngeal carcinoma; FOX: Homo sapiens forkhead box; FOXQ1: Homo sapiens forkhead box Q1; MKI67: Homo sapiens marker of proliferation Ki-67; PCNA: Homo sapiens proliferating cell nuclear antigen; TPX2: Homo sapiens TPX2, microtubule-associated; AURKA: Homo sapiens aurora kinase A; VIM: Homo sapiens vimentin; SNAI1: Homo sapiens snail family zinc finger 1; ZEB2: Homo sapiens zinc finger E-box binding homeobox 2; $\mathrm{CDH}$ 2: Homo sapiens cadherin 2, type 1, N-cadherin.

\section{Competing interests}

The authors declared that they have no competing interests.

\section{Authors' contributions}

XPL designed the experiment, interpreted the data and prepared the manuscript. XHP, HRH and $J L$ conducted the experiment, collected the data and helped to prepare the manuscript. XL, FPZ, BZ, SXL, LW, HHC, XX and FW interpreted the data. All authors read and approved the final manuscript.

\section{Acknowledgements}

This study was supported by the National Natural Science Foundation of China (U1132003, 81172053 to X.P.L.), the National Natural Science Foundation of China (81172053, to X.P.L.) and Foundation for Distinguished Young Talents in Higher Education of Guangdong, China (2012LYM_0039, to J.L.).

\section{Author details}

'Department of Otorhinolaryngology-Head and Neck Surgery, Nanfang Hospital, Southern Medical University, Guangzhou, Guangdong 510515, China. ${ }^{2}$ School of Public Health and Tropical Medicine, Southern Medical University, Guangzhou 510515, China. ${ }^{3}$ Department of Otorhinolaryngology-Head and Neck Surgery, The First Affiliated Hospital of Shantou University Medical College, Shantou, Guangdong 515041, China.

\section{Received: 5 April 2014 Accepted: 1 August 2014}

Published: 7 August 2014

\section{References}

1. Abdullah B, Alias A, Hassan S: Challenges in the management of nasopharyngeal carcinoma: a review. Malays J Med Sci 2009, 16:50-54.

2. Henderson BE: Nasopharyngeal carcinoma: present status of knowledge. Cancer Res 1974, 34:1187-1188.

3. Yoshizaki T, Ito M, Murono S, Wakisaka N, Kondo S, Endo K: Current understanding and management of nasopharyngeal carcinoma. Auris Nasus Larynx 2012, 39:137-144.

4. Chan AT: Nasopharyngeal carcinoma. Ann Oncol 2010, 21(Suppl 7):vii308-vii312.

5. Bensouda Y, Kaikani W, Ahbeddou N, Rahhali R, Jabri M, Mrabti H, Boussen $\mathrm{H}$, Errihani H: Treatment for metastatic nasopharyngeal carcinoma. Eur Ann Otorhinolaryngol Head Neck Dis 2011, 128:79-85. 
6. Lo KW, Huang DP: Genetic and epigenetic changes in nasopharyngeal carcinoma. Semin Cancer Biol 2002, 12:451-462.

7. Tao Q, Chan AT: Nasopharyngeal carcinoma: molecular pathogenesis and therapeutic developments. Expert Rev Mol Med 2007, 9:1-24

8. Chou J, Lin YC, Kim J, You L, Xu Z, He B, Jablons DM: Nasopharyngeal carcinoma-review of the molecular mechanisms of tumorigenesis. Head Neck 2008, 30:946-963.

9. Li LL, Shu XS, Wang ZH, Cao Y, Tao Q: Epigenetic disruption of cell signaling in nasopharyngeal carcinoma. Chin J Cancer 2011, 30:231-239.

10. Bartel DP: MicroRNAs: genomics, biogenesis, mechanism, and function. Cell 2004, 116:281-297.

11. Macfarlane LA, Murphy PR: MicroRNA: Biogenesis, Function and Role in Cancer. Curr Genomics 2010, 11:537-561.

12. Calin GA, Croce CM: MicroRNA signatures in human cancers. Nat Rev Cancer 2006, 6:857-866.

13. Lu J, He ML, Wang L, Chen Y, Liu X, Dong Q, Chen YC, Peng Y, Yao KT, Kung HF, Li XP: MiR-26a inhibits cell growth and tumorigenesis of nasopharyngeal carcinoma through repression of EZH2. Cancer Res 2011, 71:225-233.

14. Yu L, Lu J, Zhang B, Liu X, Wang L, Li SY, Peng XH, Xu X, Tian WD, Li XP: miR-26a inhibits invasion and metastasis of nasopharyngeal cancer by targeting EZH2. Oncol Lett 2013, 5:1223-1228.

15. Zhang LY, Ho-Fun Lee V, Wong AM, Kwong DL, Zhu YH, Dong SS, Kong KL, Chen J, Tsao SW, Guan XY, Fu L: MicroRNA-144 promotes cell proliferation, migration and invasion in nasopharyngeal carcinoma through repression of PTEN. Carcinogenesis 2013, 34:454-463.

16. Deng $M$, Ye Q, Qin Z, Zheng Y, He W, Tang H, Zhou Y, Xiong W, Zhou M, Li X, Yan Q, Ma J, Li G: miR-214 promotes tumorigenesis by targeting lactotransferrin in nasopharyngeal carcinoma. Tumour Biol 2013, 34:1793-1800.

17. Li G, Wu Z, Peng Y, Liu X, Lu J, Wang L, Pan Q, He ML, Li XP: MicroRNA-10b induced by Epstein-Barr virus-encoded latent membrane protein-1 promotes the metastasis of human nasopharyngeal carcinoma cells. Cancer Lett 2010, 299:29-36.

18. Sun XJ, Liu H, Zhang P, Zhang XD, Jiang ZW, Jiang CC: miR-10b promotes migration and invasion in nasopharyngeal carcinoma cells. Asian Pac $J$ Cancer Prev 2013, 14:5533-5537.

19. He ML, Luo MX, Lin MC, Kung HF: MicroRNAs: potential diagnostic markers and therapeutic targets for EBV-associated nasopharyngeal carcinoma. Biochim Biophys Acta 1825, 2012:1-10.

20. Lu J, Luo H, Liu X, Peng Y, Zhang B, Wang L, Xu X, Peng X, Li G, Tian W, He ML, Kung H, Li XP: miR-9 targets CXCR4 and functions as a potential tumor suppressor in nasopharyngeal carcinoma. Carcinogenesis 2014, 35 (3):554-563

21. Lu J, Xu X, Liu X, Peng Y, Zhang B, Wang L, Luo H, Peng X, Li G, Tian W, He $M, L i X$ : Predictive value of miR-9 as a potential biomarker for nasopharyngeal carcinoma metastasis. Br J Cancer 2014, 110:392-398.

22. Bieller A, Pasche B, Frank S, Glaser B, Kunz J, Witt K, Zoll B: Isolation and characterization of the human forkhead gene FOXQ1. DNA Cell Biol 2001, 20:555-561.

23. Potter CS, Peterson RL, Barth JL, Pruett ND, Jacobs DF, Kern MJ, Argraves WS, Sundberg JP, Awgulewitsch A: Evidence that the satin hair mutant gene Foxq1 is among multiple and functionally diverse regulatory targets for Hoxc13 during hair follicle differentiation. J Biol Chem 2006, 281:29245-29255.

24. Hong HK, Noveroske JK, Headon DJ, Liu T, Sy MS, Justice MJ, Chakravarti A The winged helix/forkhead transcription factor Foxq1 regulates differentiation of hair in satin mice. Genesis 2001, 29:163-171.

25. Hoggatt AM, Kriegel AM, Smith AF, Herring BP: Hepatocyte nuclear factor-3 homologue 1 ( $\mathrm{HFH}-1$ ) represses transcription of smooth muscle-specific genes. J Biol Chem 2000, 275:31162-31170.

26. Kaneda H, Arao T, Tanaka K, Tamura D, Aomatsu K, Kudo K, Sakai K, De Velasco MA, Matsumoto K, Fujita Y, Yamada Y, Tsurutani J, Okamoto I, Nakagawa K, Nishio K: FOXQ1 is overexpressed in colorectal cancer and enhances tumorigenicity and tumor growth. Cancer Res 2010, 70:2053-2063.

27. Sun $H T$, Cheng $S X, T u Y, L i X H$, Zhang S: FoxQ1 promotes glioma cells proliferation and migration by regulating NRXN3 expression. PLoS One 2013, 8:e55693.

28. Li L, Luo J, Wang B, Wang D, Xie X, Yuan L, Guo J, Xi S, Gao J, Lin X, Kong Y, Xu X, Tang H, Xie X, Liu M: Microrna-124 targets flotillin-1 to regulate proliferation and migration in breast cancer. Mol Cancer 2013, 12:163.
29. Liu K, Zhao H, Yao H, Lei S, Lei Z, Li T, Qi H: MicroRNA-124 regulates the proliferation of colorectal cancer cells by targeting iASPP. Biomed Res Int 2013, 2013:867537.

30. Lu Y, Yue X, Cui Y, Zhang J, Wang K: MicroRNA-124 suppresses growth of human hepatocellular carcinoma by targeting STAT3. Biochem Biophys Res Commun 2013, 441:873-879.

31. Xu X, Li S, Lin Y, Chen H, Hu Z, Mao Y, Wu J, Zhu Y, Zheng X, Luo J, Xie L: MicroRNA-124-3p inhibits cell migration and invasion in bladder cancer cells by targeting ROCK1. J Trans/ Med 2013, 11:276.

32. Zhang H, Wang Q, Zhao Q, Di W: MiR-124 inhibits the migration and invasion of ovarian cancer cells by targeting SphK1. J Ovarian Res 2013, 6:84.

33. Zhang J, Lu Y, Yue X, Li H, Luo X, Wang Y, Wang K, Wan J: MiR-124 suppresses growth of human colorectal cancer by inhibiting STAT3. PLOS One 2013, 8:e70300.

34. Zhao WH, Wu SQ, Zhang YD: Downregulation of miR-124 promotes the growth and invasiveness of glioblastoma cells involving upregulation of PPP1R13L. Int J Mol Med 2013, 32:101-107.

35. Wang MJ, Li Y, Wang R, Wang C, Yu YY, Yang L, Zhang Y, Zhou B, Zhou ZG, Sun XF: Downregulation of microRNA-124 is an independent prognostic factor in patients with colorectal cancer. Int J Colorectal Dis 2013, 28:183-189.

36. Liu X, Luo HN, Tian WD, Lu J, Li G, Wang L, Zhang B, Liang BJ, Peng XH, Lin SX, Peng Y, Li XP: Diagnostic and prognostic value of plasma microRNA deregulation in nasopharyngeal carcinoma. Cancer Biol Ther 2013, 14

37. Bicknell KA: Forkhead (FOX) transcription factors and the cell cycle: measurement of DNA binding by FoxO and FoxM transcription factors. Methods Mol Biol 2005, 296:247-262.

38. Zhang H, Meng F, Liu G, Zhang B, Zhu J, Wu F, Ethier SP, Miller F, Wu G: Forkhead transcription factor foxq1 promotes epithelial-mesenchymal transition and breast cancer metastasis. Cancer Res 2011, 71:1292-1301.

39. Xia L, Huang W, Tian D, Zhang L, Qi X, Chen Z, Shang X, Nie Y, Wu K: Forkhead box $\mathrm{Q} 1$ promotes hepatocellular carcinoma metastasis by transactivating ZEB2 and VersicanV1 expression. Hepatology 2014, 59:958-973.

40. Liang SH, Yan XZ, Wang BL, Jin HF, Yao LP, Li YN, Chen M, Nie YZ, Wang $X$ Guo XG, Wu KC, Ding J, Fan DM: Increased expression of FOXQ1 is a prognostic marker for patients with gastric cancer. Tumour Biol 2013, 34:2605-2609,

41. Wang W, He S, Ji J, Huang J, Zhang S, Zhang Y: The prognostic significance of FOXQ1 oncogene overexpression in human hepatocellular carcinoma. Pathol Res Pract 2013, 209:353-358.

42. Christensen J, Bentz S, Sengstag T, Shastri VP, Anderle P: FOXQ1, a novel target of the Wnt pathway and a new marker for activation of Wnt signaling in solid tumors. PLoS One 2013, 8:e60051.

43. Yu BL, Peng XH, Zhao FP, Liu X, Lu J, Wang L, Li G, Chen HH, Li XP: MicroRNA-378 functions as an onco-miR in nasopharyngeal carcinoma by repressing TOB2 expression. Int J Oncol 2014, 44:1215-1222.

44. Li XP, Li CY, Li X, Ding Y, Chan LL, Yang PH, Li G, Liu X, Lin JS, Wang J, He M, Kung HF, Lin MC, Peng Y: Inhibition of human nasopharyngeal carcinoma growth and metastasis in mice by adenovirus-associated virus-mediated expression of human endostatin. Mol Cancer Ther 2006 5:1290-1298.

doi:10.1186/1476-4598-13-186

Cite this article as: Peng et al:: MiR-124 suppresses tumor growth and metastasis by targeting Foxq1 in nasopharyngeal carcinoma. Molecular Cancer 2014 13:186. 